Cognition et sport

\title{
Effet de l'activation de stéréotypes sur le comportement : une application en contexte sportif
}

\section{Effect of stereotypes activation on behavior: an application in a sport setting}

\author{
A. Follenfant $^{\mathrm{a}, *}$, J.-B. Légal ${ }^{\mathrm{a}}$, F. Marie Dit Dinard ${ }^{\mathrm{b}}$, T. Meyer ${ }^{\text {a }}$ \\ ${ }^{a}$ Laboratoire de psychologie sociale des comportements et des cognitions, département de psychologie, EA 1588, \\ université Paris-X, 200, avenue de la République, 92001 Nanterre cedex, France \\ ${ }^{\mathrm{b}}$ Laboratoire de psychologie cognitive et pathologique, EA 1774, PPF modélisation en sciences cognitives et sociales (ModeSCoS), \\ centre de recherche sur les activités physiques et sportives, EA 2131, université de Caen Basse-Normandie, France
}

\section{Introduction}

Nous aimons croire que nos actions sont les conséquences de nos intentions. L'idée que nous avons le contrôle, que nous faisons ce que nous voulons est confortable et rassurante. Pourtant une littérature scientifique récente dans le domaine de la cognition sociale indique que le comportement ne découle pas toujours d'une intention. Ce que nous percevons dans notre environnement immédiat peut, sans que nous en ayons conscience, avoir une influence sur la façon dont nous nous comportons. Par exemple, la perception d'un individu appartenant à une catégorie donnée a toutes les chances de rendre présent à l'esprit les traits stéréotypés associés à cette catégorie. Le stéréotype, ainsi activé (i.e., rendu accessible en mémoire), peut influencer le comportement. Typique- 
ment, le fait d'activer un stéréotype conduit à se comporter de façon conforme à ce stéréotype. Par exemple, selon l'étude de Bargh et al. (1996, expérience 2), le fait d'avoir présent à l'esprit le stéréotype des personnes âgées conduit les participants à marcher plus lentement en sortant du laboratoire (que les participants du groupe témoin, cf. ci-après). De façon intéressante, il apparaît que ces effets ne sont pas conscients. Les auteurs parlent de « comportement social automatique » (« automatic social behavior », Bargh, et al., 1996).

Dans cet article, nous posons la question de la généralisation de ces effets de comportement automatique au domaine sportif. Nous nous intéressons en particulier à un comportement moteur guidé par un but de performance et réalisé, non en laboratoire, mais dans un contexte naturel. Plus précisément, nous étudierons les effets de l'activation de stéréotypes sur un comportement sportif individuel. Nous postulons que le comportement sportif peut être influencé de façon automatique par l'activation de stéréotypes pertinents au regard de l'acte sportif à accomplir.

Avant d'exposer notre étude, nous introduirons le concept de stéréotype et nous présenterons le courant de recherche sur les effets automatiques de l'activation de stéréotypes sur le comportement.

\subsection{Activation de stéréotypes}

Les stéréotypes sont des représentations mentales au sein desquelles des catégories sociales sont associées à des traits (stéréo)typiques de cette catégorie (Van Knippenberg et Dijksterhuis, 2000). Ils constituent une forme de connaissance très bien intégrée au cours de la socialisation et connue de la plupart des individus d'une société donnée (Devine, 1989). Par exemple, un stéréotype très répandu dans le domaine du sport concerne les différences de performances physiques et sportives entre athlètes noirs et athlètes blancs, les premiers étant perçus comme «plus rapides », «plus forts », « sautant plus haut, plus loin », etc. que les seconds (Stone et al., 1997). Les stéréotypes sont multicomposites dans le sens où ils sont composés à la fois de traits, de caractéristiques physiques, d'attitudes, de sentiments, d'exemplaires prototypiques et même, comme le montrent des recherches récentes, de comportements (Stangor et Lange, 1994 ; Kunda, 1999; Kawakami et al., 2002). Le recours aux stéréotypes (par exemple, « il court très vite ») présuppose une catégorisation sociale («c'est un athlète noir »). Stéréotypisation et catégorisation sont donc inséparables (pour une revue, voir De la Haye, 1998). Mais cette catégorisation initiale suffit-elle à rendre accessible en mémoire le stéréotype lié à cette catégorie ? En d'autres termes, est-ce que le simple fait d'assigner un individu à une catégorie sociale implique automatiquement l'activation des représentations stéréotypées qui lui sont associées ? La question de l'automaticité de l'activation des stéréotypes a fait l'objet d'intenses débats ces dernières années. Bon nombre de travaux sont en accord avec l'hypothèse de l'automaticité (voir notamment, Devine, 1989 ; Bargh et al., 1996 ; Bargh, 1999). Cependant, d'autres études suggèrent que les stéréo- types ne sont activés que si les ressources attentionnelles suffisantes sont disponibles (Gilbert et Hixon, 1991). De façon intéressante, Macrae et al. (1997) proposent que l'activation du stéréotype se produise de façon automatique si, et seulement si, l'information est traitée de façon sémantique, c'està-dire comme un objet ayant un sens social. Ainsi, il semble que l'activation de stéréotypes serait — selon la terminologie de Bargh — conditionnellement automatique (concept d'automaticité conditionnelle, i.e., qui ne remplit pas tous les critères de l'automaticité, voir Bargh, 1989) : l'activation automatique du stéréotype est conditionnée par le traitement sémantique de l'information perçue.

Cette activation de stéréotype peut se faire par la simple perception d'un individu appartenant à la catégorie stéréotypée (Bargh, 1994 ; Devine, 1989). L'individu perçu est catégorisé et le stéréotype associé à cette catégorie est rendu accessible. Notons cependant que dans l'étude présentée ci-après, comme dans la plupart des études sur les effets d'accessibilité, l'activation du stéréotype n'est pas induite par la perception d'un individu mais par une manipulation expérimentale permettant de rendre saillant un stéréotype donné. Il existe un grand nombre de dispositifs expérimentaux permettant d'amorcer un stéréotype. Les participants peuvent, par exemple, être exposés à des photographies d'individus appartenant à la catégorie stéréotypée ou bien à des mots traits caractéristiques de cette catégorie (via des tâches langagières ou des expositions subliminales ; Srull et Wyer, 1979 ; Bargh et Chartrand, 2000). Une autre méthode consiste à exposer les participants au nom de la catégorie (par exemple «skinhead ») et à leur demander de fournir eux-mêmes des caractéristiques applicables aux membres de la catégorie donnée (« violents », « racistes », etc.). Ces techniques d'amorçage sont mises en œuvre dans le paradigme des études indépendantes (unrelated paradigm studies) qui consiste à faire croire aux individus qu'ils participent à deux études sans aucun rapport l'une avec l'autre. La première vise à manipuler le niveau d'accessibilité d'un stéréotype donné et la seconde permet de mesurer les effets de cette manipulation à l'insu des individus. Quelle que soit la technique d'amorçage, le but est toujours d'activer tout ou partie du stéréotype. Cette activation du stéréotype correspond à une augmentation de l'accessibilité en mémoire de l'ensemble des représentations associées à une catégorie d'individus donnée. Aussi, si les stéréotypes comprennent des comportements (Kunda, 1999 ; Kawakami et al., 2002), alors ces comportements peuvent être activés à leur tour. En d'autres termes, les comportements seraient représentés mentalement, pouvant ainsi être activés en mémoire au même titre que toute autre représentation.

\subsection{Comportement automatique}

De nombreux auteurs soutiennent l'idée d'une relation étroite entre les représentations mentales et le comportement. Dès 1890, James (1890) reprenant les idées du physiologiste Carpenter (1874), évoquait la nécessité d'une connexion directe entre pensées et réponses comportementa- 
les. Selon leur principe de l'action idéomotrice (ideomotoraction), le simple fait de penser à un comportement spécifique nous amène à nous comporter nous-mêmes dans le sens de ce comportement. Autrement dit, la moindre idée à propos d'un comportement est suffisante pour augmenter la probabilité que ce comportement soit initié. James insiste aussi sur la nature passive de cet effet, argumentant qu'un acte volontaire ou intentionnel n'est pas nécessaire pour que la pensée à propos d'un comportement conduise à la réalisation de ce comportement. Récemment, Bargh et Chartrand (1999) ont repris ce principe idéomoteur en l'élargissant à la perception. La perception d'un individu appartenant à une catégorie donnée entraîne l'activation des représentations stéréotypées correspondantes, y compris les représentations comportementales. D'après les auteurs, ces représentations comportementales, une fois activées, influencent de façon automatique le comportement. Il existe aujourd'hui bon nombre de données empiriques qui démontrent que l'activation de stéréotypes affecte les comportements interpersonnels, les comportements de performance intellectuelle et les comportements moteurs. Par exemple, l'hostilité vis-à-vis d'un individu est plus importante après activation du stéréotype afro-américain (Bargh et al. 1996, expérience 3) ; les performances mnésiques sont diminuées après activation du stéréotype des personnes âgées (Dijksterhuis et al., 2000 ; Dijksterhuis, Bargh et al., 2000 ; Levy, 1996) ; les performances à une tâche de culture générale sont temporairement améliorées par l'activation du stéréotype des professeurs ou bien diminuées par l'activation du stéréotype des supporters de football (hooligans) (Dijksterhuis et Van Knippenberg, 1998) ; les performances arithmétiques peuvent être diminuées de façon transitoire par l'activation du stéréotype lié aux personnes mentalement handicapées (Follenfant et Meyer, 2001) ; etc. Parmi ces recherches sur le comportement automatique, la plus connue et aussi la plus étonnante parce qu'elle fut la première à démontrer les effets automatiques de l'activation de stéréotype sur un comportement moteur (la marche), est celle menée par Bargh et al. (1996, expérience 2). Dans cette expérience, la moitié des participants fait l'objet d'un amorçage (qui prend la forme d'une tâche de langage) avec le stéréotype des personnes âgées alors que l'autre moitié ne fait l'objet d'aucun amorçage (groupe témoin). À l'issue de cette tâche, l'expérimentateur remercie les participants, leur laissant croire que l'étude est terminée. Un compère chronomètre alors le temps mis par les participants pour parcourir la distance séparant le laboratoire de l'ascenseur. Les participants qui ont été préalablement exposés au stéréotype de la personne âgée marchent plus lentement en sortant du laboratoire que les participants du groupe témoin. Ils présentent un comportement qui correspond aux représentations les plus accessibles en mémoire. On parle d'un effet d'assimilation ou d'imitation : conformément à la représentation stéréotypée de la catégorie des personnes âgées, les participants se déplacent plus lentement (voir aussi Kawakami et al., 2000 ; Dijksterhuis et al., 2001). Les mécanismes responsables de ce type d'effets de comportement automatique sont encore mal cernés, cependant de récents travaux ouvrent des pistes intéressantes. En décomposant les actions motrices après amorçage du stéréotype des personnes âgées, Banfield et al. (2003) offrent une description plus fine des séquences motrices affectées (dans le sens d'un ralentissement, conformément au stéréotype) par le stéréotype activé.

Finalement, un large éventail de comportements peut être influencé de façon non consciente par des représentations stéréotypées. Qu'en est-il pour les comportements réalisés en contexte sportif ? Le comportement sportif est à la fois lié à des aptitudes motrices et à la poursuite de buts spécifiques. Les effets de comportement automatique ont, jusqu' alors été démontrés dans un cadre de laboratoire. Nous proposons de tester la résistance de ces effets dans un cadre naturel où des buts d'accomplissement sont en jeu et où les compétences personnelles des individus sont engagées et évaluées. Notre étude est menée sur un stade de sport auprès d'étudiants étant dans un contexte habituel de formation en sciences du sport. Nous postulons que, dans ce contexte sportif, un comportement moteur, guidé par un but spécifique de performance devrait aussi subir, à l'insu de l'individu, l'influence d'un stéréotype préalablement activé.

La seule étude, à notre connaissance, ayant démontré des effets d'activation de stéréotypes sur un comportement sportif est celle menée par Stone et al. (1999) dans le domaine de la menace de stéréotype ${ }^{1}$. Dans leurs expériences, les participants sont des étudiants masculins noirs-américains et blancs-américains qui accordent tous de l'importance aux capacités athlétiques. Ils doivent réaliser une épreuve de minigolf pour laquelle ils sont tous débutants. Pour une partie des étudiants noirs-américains la tâche de mini-golf leur est présentée comme une mesure de «l'intelligence athlétique », alors que pour une partie des étudiants blancs-américains la tâche est présentée comme une mesure « des aptitudes athlétiques naturelles ». D'autres étudiants noirs- et blancsaméricains sont affectés à une condition contrôle pour laquelle la tâche de golf ne fait pas l'objet d'une présentation particulière. Les résultats montrent que dans les deux conditions expérimentales les performances sont moins bonnes que dans les conditions de contrôle. La façon dont la tâche de minigolf est présentée aux participants noirs-américains et blancsaméricains a un effet sur leurs performances. La présentation en termes de mesure «d'aptitudes athlétiques naturelles » induit une menace pour les participants blancs qui sont alors

\footnotetext{
${ }^{1}$ Une littérature abondante sur la menace de stéréotypes démontre des effets de l'activation de stéréotypes sur des comportements variés (par exemple sur la performance en mathématiques, Spencer et al., 1999 ; sur la performance verbale, Steele et Aronson, 1995 ; ou encore sur la mémoire, Levy, 1996). Cependant, ces travaux se distinguent de ceux menés dans le champ de l'amorçage et des effets d'accessibilité par deux aspects fondamentaux : (1) le stéréotype activé est toujours applicable aux participants (i.e. les participants appartiennent à la catégorie stéréotypée) et (2) les effets du stéréotype sur le comportement sont modérés par des facteurs motivationnels (comme le niveau d'anxiété, l'appréhension de l'évaluation, etc.). (Pour une revue de question voir Croizet et Leyens, 2003 ; Croizet et al., 2003 ; Steele et al., 2002).
} 
moins performants que le groupe témoin. À l'inverse, la présentation de la tâche en tant que mesure «d'intelligence athlétique » présente une menace pour les participants noirs qui réalisent alors de moins bonnes performances que le groupe témoin (bien sûr, la tâche de mini-golf n'était en fait ni une mesure de l'intelligence ni une mesure d'aptitudes athlétiques naturelles). Les auteurs interprètent ces effets d'après la théorie de la menace de stéréotype (Stereotype Threat Theory, Steele et Aronson, 1995 ; Croizet et Leyens, 2003). Lorsqu' un stéréotype négatif est activé (ici par le mode de présentation de la tâche de mini-golf) les participants se sentent menacés par ce stéréotype et leurs performances s'en trouvent diminuées. Les effets seraient donc dus au fait que les participants appartiennent au groupe stéréotypé et qu'ils se sentent ainsi menacés par la présentation stéréotypée de la tâche.

Une originalité de notre étude est de tester l'hypothèse d'un lien direct entre l'activation de stéréotype et le comportement sportif. Il ne s'agit donc pas de menacer l'identité des participants, mais de les exposer à une catégorie stéréotypée à laquelle ils n'appartiennent pas. Les stéréotypes ne sont alors pas choisis pour leur degré d'applicabilité quant aux participants, mais pour leur applicabilité au comportement sportif mesuré (Higgins, 1996). Ce comportement sportif est réalisé dans un contexte naturel d'évaluation, ainsi les participants poursuivent un but de performance. Nous avons sélectionné une activité sportive qui se pratique de façon individuelle, qui puisse être mesurée quantitativement et qui ne nécessite pas ou peu d'apprentissage. De plus, afin de maximiser la validité écologique de l'étude, nous avons choisi une activité sportive existante dans le cadre pédagogique de notre intervention. Parmi les différents ateliers d'athlétisme suivis par les étudiants, nous avons sélectionné celui de lancer de balle lestée pour lequel il s'agit de lancer, sans prise d'élan, une balle de $500 \mathrm{~g}$ le plus loin possible dans l'aire de lancer. Cet exercice exige à la fois des aptitudes de conditions physiques (force explosive, etc.) et de coordination (timing de lâcher de la balle, cf. Hore et al., 1995).

\subsection{Hypothèse}

Nous postulons que les performances de lancer de balle lestée sont influencées par le stéréotype préalablement activé. De façon conforme avec la théorie sur les effets automatiques sur le comportement, nous anticipons des effets d'assimilation, c'est-à-dire une influence dans un sens congruent avec le contenu du stéréotype. Plus précisément, nous attendons une amélioration des performances de lancer chez les participants préalablement exposés au stéréotype des basketteurs et une détérioration de celles-ci chez les participants exposés au stéréotype des personnes âgées.

\section{Méthode}

\section{1. Étude pilote}

Pour qu'un stéréotype exerce une influence sur un comportement donné, ce stéréotype doit être applicable au com- portement (voir Higgins, 1996 pour un point complet sur cette notion d'applicabilité). Bien que les effets se déroulent à l'insu des individus, ils vont tout de même dans le sens de leurs croyances générales. Pour être applicable, le stéréotype doit être pertinent par rapport au comportement mesuré ou en tout cas perçu comme tel par les individus. Ainsi, une fois le comportement sportif sélectionné, une étude pilote a été nécessaire afin de déterminer les stéréotypes applicables à ce comportement spécifique de lancer de balle lestée. Assumant que les effets sur le comportement sont inconscients et non intentionnels, il nous semblait important de retenir un stéréotype lié à de bonnes performances ainsi qu'un stéréotype lié à de mauvaises performances en lancer de balle. Si l'amélioration de la performance est positive et désirable, sa diminution apparaît comme négative et non désirable. Ainsi, le fait de démontrer des effets non désirables sur le comportement atteste de la nature automatique des effets (cf. Bargh et al. 1996 ; Dijksterhuis et Van Knippenberg, 1998), simplement parce que les individus ne vont pas intentionnellement s'engager dans un comportement indésirable (e.g., obtenir une mauvaise performance). Afin de déterminer les catégories d'individus étant perçus par notre population de sportifs comme les plus vs. les moins performants dans cette activité de lancer de balle lestée, nous avons mené une étude pilote auprès de 25 étudiants en sciences du sport. Un questionnaire proposait une trentaine de catégories de sportifs (e.g. haltérophiles, rugbymen, etc.) et de non sportifs (e.g., handicapés, personnes âgées, etc.). Les participants estimaient, pour chacune de ces catégories, les performances de lancer au moyen d'une échelle de type Likert (de $1:$ «très mauvaises » à $9:$ : excellentes »). Il était spécifiquement indiqué aux participants que la performance de lancer correspondait à lancer une balle lestée le plus loin possible ( plus l'individu lance la balle loin, plus on considère qu'il est performant »). Deux catégories d'individus se différenciant fortement sur cette dimension de lancer de balle ont été retenues pour l'étude : la catégorie des «basketteurs » dans le sens d'une performance élevée et celle des « personnes âgées » dans le sens d'une performance faible en lancer de balle, $M s=7,16$ et 2,44; ETs $=1,11$ et 1,18, $t(1,48)=14,56 ; p<0,0001$.

Finalement, de façon stéréotypée, les basketteurs sont perçus par notre population d'étudiants en sport comme des individus très compétents pour lancer une balle lestée très loin et, a contrario, les personnes âgées sont perçues comme incompétentes pour cette tâche de lancer.

\subsection{Participants et Plan expérimental}

Du fait d'importantes différences liées au genre dans ce type de performance physique nous avons choisi de ne retenir que des participants de sexe masculin. Les participants $(N=48)$ sont des étudiants en première année de sciences du sport dans une université parisienne. Ils ont été affectés, de manière aléatoire, à l'amorçage du stéréotype des personnes âgées ou du stéréotype des basketteurs. Tous les étudiants ont accepté de participer à cette étude et aucun d'entre eux n'a 
déclaré pratiquer le basket ou une activité de lancer (poids, javelot, etc.) à un niveau de compétition ou de façon régulière. Ils connaissent tous cette activité de lancer de balle lestée pour l'avoir déjà pratiquée durant quelques séances d'entraînement. On peut donc considérer qu'ils ont un niveau d'expertise équivalent dans cette activité spécifique.

\subsection{Terrain et Comportement Sportif}

L'expérience est menée sur un stade de sport à l'occasion d'un cours d'athlétisme comprenant plusieurs ateliers d'entraînement à diverses activités physiques. Celui retenu pour cette étude est l'atelier de lancer de balle lestée, pour lequel il s'agit de lancer, sans prise d'élan, une balle de $500 \mathrm{~g}$ le plus loin possible. Le comportement mesuré est la performance, en mètres et centimètres, de lancer. Deux prises de performances sont effectuées à une semaine d'intervalle : une avant l'activation de stéréotypes (pré-test) et une seconde après activation de stéréotypes (post-test). Pour chacune de ces deux prises de performance, les étudiants sont informés qu'il s'agit d'une évaluation : ils poursuivent alors tous, de façon consciente, un but de performance (i.e., lancer le plus loin possible).

\subsection{Procédure}

Un pré-test a été réalisé une semaine avant le test, du fait d'une grande variabilité des performances entre les étudiants dans cette activité de lancer. Ce prétest permet de contrôler la part de variabilité interindividuelle en référençant la mesure de la performance à l'individu lui-même ([self-referenced], Lane et al., 2001). Les performances au prétest ont été relevées par les enseignants une semaine avant que les expérimentateurs ne viennent présenter l'étude sur le stade de sport. Le protocole de recueil de performances avant manipulation s'est déroulé de manière standardisée de façon à ce qu'il puisse être reproduit lors du relevé des performances après manipulation. Les étudiants devaient réaliser deux lancers consécutifs sans prise d'élan. Ils étaient informés que leur meilleure performance (i.e., le lancer le plus long) serait retenue pour leur évaluation pédagogique finale (implémentant ainsi la poursuite d'un but conscient de performance). Une semaine plus tard, deux expérimentateurs se sont présentés avec chacun une étude à leur proposer. La première, présentée comme une étude sur « l'influence du contexte sur la mémoire des personnes », vise à activer le stéréotype des personnes âgées vs. des basketteurs. La seconde étude, annoncée comme une étude sur «l'autoévaluation de performances sportives », sert à justifier le relevé de performance et à vérifier que les participants n'ont pas établi de lien entre les deux études présentées (phase d'activation de stéréotype et phase de lancer). Conformément au paradigme des études indépendantes, les étudiants croient donc participer à deux études distinctes.

En résumé, les participants effectuent un premier lancer une semaine avant l'étude afin d'établir une mesure indivi- duelle de référence (pré-test). Le jour de l'étude, (1) ils remplissent le questionnaire d'amorçage sur les personnes âgées ou les basketteurs pendant trois minutes, (2) ils effectuent ensuite leur épreuve de lancer (dans les mêmes conditions que lors du pré-test), et enfin (3) ils répondent au questionnaire postexpérimental sur l'éventuelle perception d'un lien entre l'amorçage de stéréotype et la performance en lancer. En fin de séance, tous les participants sont remerciés et débriefés.

\subsection{Matériel et technique d'amorçage}

La technique d'amorçage utilisée pour cette étude prend la forme d'une tâche de production écrite de type association libre. Les participants doivent fournir le plus grand nombre possible de caractéristiques (traits, adjectifs, exemplaires typiques, etc.) associées à une catégorie donnée (personnes âgées vs. basketteurs) en un temps limité. Pour cela ils disposent d'une feuille sur laquelle sont présentées la consigne ainsi qu'une vingtaine de phrases à compléter de type « Les personnes âgées sont ... » ou « Les basketteurs sont ... ». Les participants font donc l'objet d'un double amorçage. Ils sont exposés de manière répétée à une catégorie dont le nom est inscrit 20 fois sur le document (amorçage par exposition répétée) et ils doivent générer eux-mêmes des mots-traits associés à cette catégorie (amorçage par production). Les participants sont limités dans le temps : ils sont interrompus au bout de trois minutes et vont directement sur l'aire de lancer. L'expérimentateur en charge de la phase d'amorçage n'était jamais présent sur l'aire de lancer. Le relevé de performance était effectué par un enseignant (possédant l'expertise de ce type de mesure) aveugle quant aux conditions expérimentales.

Enfin, un questionnaire postexpérimental permettait de vérifier que les participants n'établissaient pas de lien entre l'activation du stéréotype et leur performance de lancer (inspiré du « questionnaire en entonnoir »[funneled questionnaire], Bargh et Chartrand, 2000).

\section{Résultats}

\subsection{Contrôle des manipulations}

Les réponses des participants au questionnaire postexpérimental indiquent qu'aucun des participants n'a explicitement suspecté le lien entre la phase d'activation de stéréotype et les performances en lancer. Les données des 48 participants ont donc été conservées pour les analyses.

\subsection{Performance en lancer de balle lestée}

Les données ont été traitées selon une analyse de variance mixte avec les performances au pré-test et au post-test en mesures répétées et l'amorçage en facteur indépendant. Cette ANOVA révèle une interaction significative entre les deux 
facteurs, $F(1,46)=4,99, p<0.05$. Les différences de performances entre les deux lancers sont fonction de la condition d'amorçage. La différence de performance au post-test entre les deux groupes de participants est d'environ 1,8 m dans le sens attendu : les participants chez qui le stéréotype des personnes âgées a été préalablement amorcé ont lancé moins loin que ceux chez qui le stéréotype des basketteurs avait été amorcé. Par rapport à la performance moyenne obtenue au pré-test, le pattern de résultats va bien dans le sens attendu que ce soit pour l'amorce « personnes âgées » $(M s=35,8$ et $34,6, E T s=7,2$ et $7,4, n s)$ comme pour l'amorce « basketteurs » $(M s=34,8$ et 36,4, ET = 6,4 et 5,6, $p<0,09$, test posthoc LSD) cependant les analyses univariées sont en deçà du seuil de significativité.

Afin de rendre compte de la différence de performance entre le $1^{\text {er }}$ et le $2^{\text {nd }}$ lancer, un score correspondant au pourcentage de fluctuation intra-individuelle de la performance (qui permet une meilleure mesure de la performance sportive, Beedie et al., 2000) a été calculé pour chaque participant : [(Perf. 2 - Perf. 1)/Perf. 1] x 100. L'ANOVA sur ces scores montre également un effet significatif du stéréotype dans le sens attendu, $F(1,46)=5,11, p<0,03$; cf. Fig. 1 . L'activation du stéréotype lié aux personnes âgées a produit une diminution de la performance de $2,7 \%$ en moyenne $(E T=12,87)$. A l'inverse, l'activation du stéréotype lié aux basketteurs a provoqué une amélioration moyenne de 5,8 \% $(E T=13,08)$ de la performance en lancer. On note une asymétrie selon la condition d'amorçage : l'effet d'amélioration de la performance consécutif à l'amorçage du stéréotype des basketteurs est plus «fort » que celui de diminution de la performance suite à l'amorçage du stéréotype des personnes âgées ${ }^{2}$.

De façon intéressante, la mesure des performances de lancer en mesures répétées (pré-test et post-test) permet d'évaluer la validité individuelle des effets ${ }^{3}$. L'analyse des données en termes de fréquence montre que parmi les participants exposés à l'amorce « personnes âgées » $(n=27), 67 \%$ d'entre eux ont vu leur performance diminuer entre les deux lancers.

Ces derniers ont perdu en moyenne $3,4 \mathrm{~m}$ entre les deux lancers $(n=18 ; E T=3,23)$. Alors que $33 \%$ d'entre eux ont amélioré leur performance entre le prétest et le post-test (amélioration moyenne de 3,3 m, $n=9 ; E T=2,98)$. A l'inverse, on observe que parmi les participants de la condition d'amorçage « basketteurs » $(n=21), 62 \%$ ont amélioré leur performance (de $3,9 \mathrm{~m}$ en moyenne ; $n=13 ; E T=2,77$ ). Alors que les $38 \%$ restant ont vu leur performance diminuer de $2,26 \mathrm{~m}$ en moyenne $(n=8 ; E T=1,13)$. Il y a donc plus de participants qui ont amélioré leur performance lorsqu'ils ont préa-

\footnotetext{
${ }^{2}$ Cette asymétrie est probablement due, en partie, à la mesure répétée. Le fait de répéter la mesure a généralement un effet facilitateur sur la performance, du fait d'un apprentissage (du geste, de la situation, etc.). Cet effet de la mesure, en interaction avec l'effet d'amorçage, viendrait donc accentuer l'effet de l'amorce « basketteurs » sur la performance, alors qu'à l'inverse il devrait réduire l'effet de l'amorce «personnes âgées ».

${ }^{3}$ Nous remercions un expert anonyme pour nous avoir suggéré cette exploitation complémentaire des données.
}

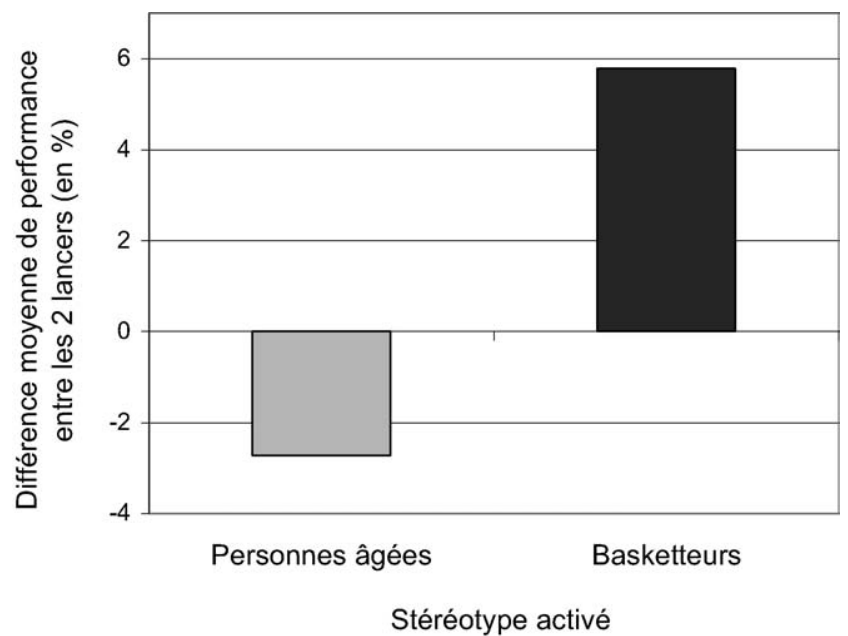

Fig. 1. Différence moyenne de performance (en \%) entre le $1^{\mathrm{er}}$ lancer (prétest) et le $2^{\text {nd }}$ lancer (post-test) selon le stéréotype activé.

Un score positif indique une amélioration de la performance et un score négatif indique une diminution de la performance entre le pré-test et le posttest.

lablement été exposés au stéréotype des basketteurs, à l'inverse ils sont plus nombreux à voir leur performance diminuer lorsqu'ils ont activé le stéréotype des personnes âgées, $\chi 2=3,88, \mathrm{dl}=1, p<0,05$.

\section{Discussion et conclusion}

Le but de cette expérience était de montrer que l'activation de stéréotypes peut avoir une influence directe sur un comportement sportif dans un contexte où l'individu recherche intentionnellement la performance. Les données obtenues dans un contexte sportif naturel vont bien dans le sens d'un effet de l'activation de stéréotypes sur une performance sportive individuelle. Il apparaît que la performance de lancer est fonction du stéréotype préalablement activé en mémoire (basketteurs ou personnes âgées). De façon conforme à nos attentes, cet effet va dans le sens d'une assimilation (ou imitation, Bargh et Chartrand, 1999) : l'exposition à une catégorie stéréotypée influence la performance sportive dans un sens congruent avec le contenu du stéréotype. Alors que l'activation du stéréotype des personnes âgées conduit à une détérioration de la performance, l'activation du stéréotype des basketteurs améliore cette dernière. À l'inverse de l'étude de Stone et al. (1999), les stéréotypes activés ne sont pas, ici, directement applicables aux participants euxmêmes. Autrement dit, les participants n'appartiennent pas aux catégories activées (basketteurs ou personnes âgées). Aussi, l'interprétation en termes de menace de l'identité n'est pas appropriée. En revanche, les effets sont conformes à la théorie du comportement automatique : l'activation de stéréotypes influence - de façon non-consciente et nonintentionnelle - le comportement subséquent. Les données accréditent l'idée d'une relation directe entre la perception et le comportement et permettent de généraliser ces effets automatiques à un comportement sportif. De futures recherches 
sont encore nécessaires notamment afin de déterminer clairement quels sont les mécanismes par lesquels la perception d'un individu ou d'un groupe d'individus influence le comportement sportif. D'après les travaux de Kawakami et al. (2002), on peut penser que l'activation du stéréotype a conduit à activer en mémoire tout un ensemble d'éléments qui lui sont associés (i.e., nœud d'activation), et parmi ceux-ci des représentations comportementales (Kunda, 1999). Une fois ces représentations comportementales activées en mémoire, l'individu aurait tendance à se comporter lui-même en accord avec ces représentations (Bargh et Chartrand, 1999). De cette façon, si le comportement mesuré est cognitivement lié au stéréotype activé, alors il serait activé à son tour. Dans notre étude, ce lien entre les stéréotypes et le comportement spécifique de lancer n'a pas été mesuré chez les participants. Il a cependant fait l'objet d'une étude pilote auprès d'un échantillon issu de la même population que les participants (i.e., étudiants en sciences du sport). On peut donc présumer que les participants associent le stéréotype des basketteurs à de bonnes performances en lancer et celui des personnes âgées à de mauvaises performances en lancer. Il nous semble pourtant peu probable que le comportement spécifique de lancer de balle lestée soit représenté et directement lié à la catégorie des basketteurs et - d'autant moins probable encore - à celle des personnes âgées. Nous proposons que le nœud activé en mémoire par l'amorçage de stéréotype n'est pas lié à un comportement spécifique mais plutôt à une tendance comportementale générale. Par exemple, l'activation du stéréotype des personnes âgées activerait non pas un ensemble de comportements spécifiques, mais une tendance comportementale plus générale de l'ordre du ralentissement moteur, de la diminution physique, etc. Une variété importante de comportements pourrait ainsi être influencée directement par l'activation du stéréotype des personnes âgées : la vitesse de marche (Bargh et al., 1996), le temps de réponse lors d'une tâche de décision (Kawakami et al. 2000) ou encore la performance sportive de lancer. De façon similaire, le stéréotype des basketteurs n'activerait pas directement le comportement spécifique de « lancer loin », mais une tendance comportementale générale (bonnes conditions physique et sportive, aptitudes athlétiques, etc.) qui guiderait le comportement dans ce sens. Notons que, selon cette interprétation, l'association cognitive entre le stéréotype activé en mémoire et le comportement spécifique mesuré paraît susceptible de moduler fortement les effets. Il serait donc particulièrement intéressant, pour les recherches à venir, d'avoir une mesure implicite de cette association chez les participants eux-mêmes ainsi qu'une mesure de la force de cette association cognitive (i.e., dans quelle mesure chacun des participants associe le comportement mesuré au stéréotype considéré). On peut se demander si les représentations comportementales activées suite à l'exposition aux stéréotypes des basketteurs vs. des personnes âgées sont équivalentes. Il est probable que le stéréotype des basketteurs active des représentations plus applicables à la tâche de lancer que le stéréotype des personnes âgées. La représentation stéréotypée des basketteurs active peut-être des images motrices plus pro- ches et plus pertinentes par rapport à l'épreuve de lancer, donc plus susceptibles d'influencer le comportement subséquent.

Ces premiers résultats sur l'effet des stéréotypes sur le comportement en contexte sportif ont des implications dans le domaine du sport qui nous semblent non négligeables. Ils permettent notamment une meilleure connaissance des facteurs pouvant influencer la performance dans un cadre sportif, où le succès dépend largement des performances motrices individuelles et de la capacité à poursuivre un but spécifique. Le fait de penser à une catégorie d'individus que l'on associe à des aptitudes physiques et sportives diminuées ou excellentes peut avoir des conséquences congruentes sur un comportement sportif. Le comportement suit la pensée, sans même que nous n'en ayons conscience. Ce lien entre les représentations mentales (ou pensées) et le comportement est déjà bien connu des entraîneurs sportifs et des athlètes qui utilisent fréquemment des méthodes d'imagerie mentale (Ungerleider et Golding, 1992). Il semble que les athlètes possèdent une connaissance intuitive de ce type d'effet. L'imagerie mentale est une stratégie cognitive qui consiste à évoquer les caractéristiques d'un objet, événement ou processus absent de notre champ perceptif actuel, que celui-ci appartienne au passé, au présent ou au futur (Denis, 1985 ; Murphy et Jowdy, 1992). Aussi, on peut observer une certaine analogie entre ce type de pratiques et les effets de comportement automatique. Par exemple, Gould et al. (1980) montrent que le recours à des stratégies d'imagerie ou d'activation a des effets significativement positifs sur la performance à une tâche d'haltérophilie. D'autres auteurs (Woolfolk et al., 1985) ont montré que l'imagerie positive améliore les performances en golf, alors qu'une imagerie négative les détériore. Le recours croissant à des techniques d'entraînement mental dans le domaine du sport, est corroboré aujourd' hui par de récentes études en psychologie et neurophysiologie. Il apparaît, dans notre étude, que les représentations qui sont les plus accessibles en mémoire au moment de réaliser l'acte sportif de lancer influencent la performance. De nombreux travaux ont cherché à déterminer si les représentations mentales entretiennent un lien avec des réactions physiologiques et/ou musculaires. Le fait d'imaginer des actions motrices complexes (telles que courir, ramer, lever des poids, etc.) entraîne les mêmes modifications neurophysiologiques (activation neuronale, fréquence cardiaque, etc.) que la réalisation réelle de ces actions (Decety et al., 1991 ; voire aussi le phénomène d'isochronie, Decety et al., 1989). Autrement dit, ce que nous avons en tête a déjà des conséquences aux niveaux physiologique et comportemental. De la même façon, si le stéréotype active des représentations comportementales, alors il est très probable que ces représentations aient des conséquences au niveau du comportement. Les résultats de notre étude vont dans ce sens. Bien sûr, cette étude ne constitue qu'un premier pas dans l'application des ces effets automatiques au comportement sportif. De futures recherches devraient considérer d'autres comportements sportifs afin de généraliser ces effets, et envisager des mesures supplémentaires (mesures physiologiques, mesures implicites d'association cognitive, etc.) pour mieux cerner les mécanismes sous-jacents. 


\section{Conclusion}

Cette étude montre que le fait que tel ou tel stéréotype soit actif au moment de réaliser une performance sportive n'est pas sans conséquence sur l'issue de celle-ci. Le stéréotype accessible peut avoir des effets facilitateurs ou inhibiteurs sur la performance sportive. Dans un contexte d'évaluation sportive où le but explicite poursuivi par les individus est de réaliser une bonne performance (ici, lancer le plus loin possible), le stéréotype préalablement activé interviendrait comme une consigne implicite guidant le comportement. Cette consigne implicite pourrait alors interférer avec la consigne explicite de performance. Une des limites de notre étude est de ne pas permettre l'exploration de cette interaction entre buts explicite et implicite, puisque l'ensemble de nos participants poursuit un but explicite de performance. Pourtant, il nous semble que cette étude ouvre des pistes intéressantes pour de futures recherches et nous nous employons à préciser les effets de compatibilité vs. d'incompatibilité de buts implémentés par une consigne explicite ou implicite (Légal et al., 2005). Dans le cadre spécifique du sport, on peut poser la question de la robustesse de ces effets en fonction des buts instaurés par la situation sportive elle-même (par exemple, entraînement vs. compétition), préjugeant que la pression à la performance est susceptible de moduler ces effets.

\section{Remerciements}

Nous remercions l'équipe pédagogique de l'UFR STAPS de l'Université Paris-X d'avoir rendu possible cette étude.

\section{Références}

Banfield, J.F., Pendry, L.F., Mewse, A.J., Edwards, M.G., 2003. The effects of an elderly stereotype prime on reaching and grasping actions. Soc. Cogn. 21 (4), 299-319.

Bargh, J.A., 1989. Conditional automaticity: Varieties of automatic influence in social perception and cognition. In: Uleman, J.S., Bargh, J.A. (Eds.), Unintended thoughts (pp. 3-51). The Guilford Press, NY.

Bargh, J.A., 1994. The Four Horsemen of Automaticity. In: Wyer, R.S., Srull, T.K. (Eds.), Handbook of social cognition. Erlbaum, Hillsdale, NJ, pp. 1-40.

Bargh, J.A., 1999. The cognitive monster: The case against the controllability of automatic stereotype effects. In: Chaiken, S., Trope, Y. (Eds.), Dual process theories in social psychology. Guilford, New York.

Bargh, J.A., Chartrand, T.L., 1999. The unbearable automaticity of being. Am. Psychol. 54, 462-479.

Bargh, J.A., Chartrand, T.L., 2000. The mind in the middle: A practical guide to priming ans automaticity research. In: Reis, H., Judd, C. (Eds.), Handbook of research methods in social and personality psychology. Cambridge University Press, New York, pp. 253-285.

Bargh, J.A., Chen, M., Burrows, L., 1996. Automaticity of social behavior: Direct effects of trait construct and stereotype priming on action. J. Pers. Soc. Psychol. 71, 230-244.

Beedie, C.J., Terry, P.C., Lane, A.M., 2000. The profile of mood states and athletic performance: two meta-analyses. J. Appl. Sport Psychol. 12, 49-68.
Carpenter, W.B., 1874. Principles of mental physiology. Appleton, New York.

Croizet, J.-C., Désert, M., Dutrévis, M., Leyens, J.-P., 2003. L’impact des réputations d'infériorité sur les performances intellectuelles. Revue Internationale de Psychologie Sociale 16, 97-124.

Croizet, J.C., Leyens, J.Ph., 2003. Mauvaises réputations : réalités et enjeux de la stigmatisation sociale. Armand Colin, Paris.

Decety, J., Jeannerod, M., Germain, M., Pastenne, J., 1991. Vegetative response during imagined movement is proportional to mental effort. Behav. Brain Res. 42, 1-5.

Decety, J., Jeannerod, M., Prablanc, C., 1989. The timing of mentally represented actions. Behav. Brain Res. 34, 35-42.

De la Haye, A.M., 1998. La catégorisation des personnes. Presses Universitaires de Grenoble, Grenoble.

Denis, M., 1985. Visual imagery and the use of mental practice in the development of motor skills. Can. J. Appl. Sport Sci. 10, 4S-16S.

Devine, P., 1989. Stereotypes and prejudice: Their automatic and controlled components. J. Pers. Soc. Psychol. 56, 680-690.

Dijksterhuis, A., Aarts, H., Bargh, J.A., Van Knippenberg, A., 2000. On the relation between associative strength and automatic behavior. J. Exp. Soc. Psychol. 36, 531-544.

Dijksterhuis, A., Bargh, J.A., Miedema, J., 2000. Of men and mackerels: Attention and automatic behavior. In: Bless, H., Forgas, J.P. (Eds.), Subjective experience in social cognition and behavior. Psychology Press, Philadelphia, pp. 36-51.

Dijksterhuis, A., Spears, R., Lepinasse, V., 2001. Reflecting and deflecting stereotypes: Assimilation and contrast in impression formation and automatic behavior. J. Exp. Soc. Psychol. 37, 286-299.

Dijksterhuis, A., Van Knippenberg, A., 1998. The relation between perception and behavior, or how to win a game of Trivial Pursuit. J. Pers. Soc. Psychol. 74, 865-877.

Follenfant, A., Meyer, T. (2001, juin). Induire un déficit de performances par la suppression de stéréotypes associés aux capacités cognitives. Papier présenté au Cinquième Colloque Jeunes Chercheurs en Psychologie Sociale de l'Association pour la Diffusion de la Recherche Internationale en Psychologie Sociale, Aix-en-Provence, France.

Gilbert, D.T., Hixon, J.G., 1991. The trouble of thinking: Activation and application of stereotypic beliefs. J. Pers. Soc. Psychol. 60, 509-517.

Gould, D., Weinberg, R.S., Jackson, A., 1980. Mental preparation strategies, cognitions, and strength performance. Journal of Sport Psychology 2, 329-339.

Higgins, E.T., 1996. Knowledge activation: Accessibility, applicability and salience. In: Higgins, E.T., Kruglanski, A.W. (Eds.), Social Psychology: Handbook of basic principles. Guilford Press, New York.

Hore, J., Watts, S., Martin, J., Miller, B., 1995. Timing of finger opening and ball release in fast and accurate overarm throws. Exp. Brain Res. 103, 277-286.

James, W., 1890. Principles of psychology. Holt, New York.

Kawakami, K., Young, H., Dovidio, J.F., 2002. Automatic Stereotyping: Category, Trait, and Behavioral Activations. Pers. Soc. Psychol. Bull. 28, $3-15$.

Kawakami, K., Young, H., Dovidio, J.F., Dijksterhuis, 2000. Effect of social category priming on personal attitudes. Psychological Science 14, 313 319.

Kunda, Z., 1999. Social cognition: Making sense of people. MIT Press, Cambridge, MA.

Lane, A.M., Terry, P.C., Beedie, C.J., Curry, D.A., Clark, N., 2001. Mood and performance: test of a conceptual model with a focus on depressed mood. Psychol. Sport Exerc. 2, 157-172.

Légal, J.-B., Delouvée, S., Follenfant, A., Meyer, T., 2005. Nonconscious and Conscious Goal Pursuit: The case of compatibility or incompatibility between goals (in press).

Levy, B., 1996. Improving memory in old age by implicit self-stereotyping. J. Pers. Soc. Psychol. 71, 1092-1107.

Macrae, C.N., Bodenhausen, G.V., Milne, A.B., Thorn, T.M.J., Castelli, L., 1997. On the activation of social stereotypes: The moderating role of processing objectives. J. Exp. Soc. Psychol. 33 (5), 471-489. 
Murphy, S.M., Jowdy, D.P., 1992. Imagery and mental practice. In: Horn, T.S. (Ed.), Advances in Sport Psychology. Human Kinetics, Champaign, IL, pp. 221-250.

Spencer, S., Steele, C.M., Quinn, D., 1999. Under suspicion of inability: Stereotype threat and women's math performance. J. Exp. Soc. Psychol. $37,1660-1672$.

Srull, T.K., Wyer Jr., R.S., 1979. The role of category accessibility in the interpretation of information about persons: Some determinants and implications. J. Pers. Soc. Psychol. 37, 1660-1672.

Stangor, C., Lange, J., 1994. Mental representations of social groups: Advances in understanding stereotypes and stereotyping. In: Zanna, M. (Ed.), Advances in Experimental Social Psychology, Vol. 25. Academic Press, New York, pp. 357-416.

Steele, C.M., Aronson, J., 1995. Stereotype threat and the intellectual test performance of Africans Americans. J. Pers. Soc. Psychol. 69, 797-811.

Steele, C.M., Spencer, S.J., Aronson, J., 2002. Contending with bias: The psychology of stereotype and social identity threat. In: Zanna, M.P.
(Ed.), Advances in Experimental Social Psychology, vol. 34. Academic Press, San Diego, pp. 277-341.

Stone, J., Perry, Z.W., Darley, J.M., 1997. "White men can't jump": Evidence for the perceptual confirmation of racial stereotypes following a basketball game. Basic Appl. Soc. Psych. 19, 291-306.

Stone, J., Lynch, C.I., Sjomeling, M., Darley, J.M., 1999. Stereotype threat effects on black and white athletic performance. J. Pers. Soc. Psychol. $77,1213-1227$.

Ungerleider, S., Golding, J.M., 1992. Beyond strength: Psychological profiles of Olympic athletes. Wm. C. Brown, Dubuque, IA.

Van Knippenberg, A., Dijksterhuis, A., 2000. Social categorisation and stereotyping: A functional perspective. In: Stroebe, W., Hewstone, M. (Eds.), European Review of Social Psychology, Vol. 11. Wiley, Chichester, pp. 105-144.

Woolfolk, R., Parrish, W., Murphy, S.M., 1985. The effects of positive and negative imagery on motor skill performance. Cognit. Ther. Res. 9, $235-341$. 